\title{
TRADUÇÃO DO TEXTO DE WALTER BENJAMIN "A TAREFA DO TRADUTOR" PARA A LÍNGUA BRASILEIRA DE SINAIS, A PARTIR DA TRADUÇÃO DE SUSANA KAMPFF LAGES
}

\author{
Ádila Silva Araújo Marques \\ Kátia Lucy Pinheiro \\ Thaís Fleury Avelar \\ Universidade Federal de Santa Catarina \\ Florianópolis, Santa Catarina, Brasil
}

Resumo: Este artigo apresenta uma tradução comentada para a Língua Brasileira de Sinais (Libras), disponibilizada em vídeo, do texto de Walter Benjamin "A Tarefa do Tradutor" - extraído da série Clássicos da Teoria da Tradução (antologia bilíngue, alemão-português, 2010) -, a partir da tradução de Susana Kampff Lages.

Palavras-chave: Teoria da Tradução. Tradução comentada. Libras.

\section{A BRAZILIAN SIGN LANGUAGE TRANSLATION OF SUSANA KAMPFF LAGES' TRANSLATION OF WALTER BENJAMIN'S TEXT "THE TASK OF THE TRANSLATOR"}

\begin{abstract}
This article presents a commented translation in Brazilian Sign Language (Libras), available in video, of Walter Benjamin's "The Task of the Translator", which has been taken from the Classics of Translation Theory series (a 2010 bilingual anthology in German and Portuguese) based on a translation by Susana Kampff Lages.
\end{abstract}

Keywords: Translation theory. Commented translation. Libras. 


\section{Referência}

BENJAMIN, Walter. A Tarefa do Tradutor. Tradução de Susana Kampff Lages. In: HEIDERMANN, Werner (Org.). Clássicos da teoria da tradução, v. 1, Alemão-Português, $2^{\text {a }}$ ed. revisada e ampliada. Florianópolis: UFSC/Núcleo de Pesquisas em Literatura e Tradução, 2010, pp. 201-231. Antologia bilíngue.

Recebido em: 10 de outubro de 2017 Aceito em: 30 de janeiro de 2018 Publicado em: maio de 2018

Ádila Silva Araújo Marques. E-mail: adila.tils@gmail.com

Kátia Lucy Pinheiro. E-mail: katialucyp@gmail.com

Thaís Fleury Avelar. E-mail: thaisfleury13@gmail.com 